\title{
PMIPv6 Assistive Cross-Layer Design to reduce handover latency in VANET Mobility for Next Generation Wireless Networks
}

\author{
Haider N. Hussain AL-Hashimi \\ Dep. Of Computer Science, Faculty of Education for Pure Science \\ Basra University, Basra (Iraq) \\ E-mail: alhashmiy@gmail.com
}

Walled N. Hussein

Dep. Of Computer Engineering, Iraq University College (Basra, Iraq)

E-mail: waleedn9@yahoo.com

Received: July 1, 2015 Accepted: October 9, 2015 Published: November 28, 2015

DOI: 10.5296/npa.v7i3.7943

URL: http://dx.doi.org/10.5296/npa.v7i3.7943

\begin{abstract}
VANETs are one of the main next generation wireless networks which are envisaged to be an integration of homogeneous and heterogeneous wireless networks. The inter-networking of these wireless networks with the Internet will provide ubiquitous access to roaming network users. However, a seamless handover mechanism with negligible handover delay is required to maintain active connections during roaming across these networks. Several solutions, mainly involving host-based localized mobility management schemes, have been widely proposed to reduce handover delay among homogeneous and heterogeneous wireless networks. However, the handover delay remains high and unacceptable for delay-sensitive services such as real-time and multimedia services. Moreover, these services will be very common in next generation wireless networks. Unfortunately, these widely proposed host-based localized mobility management schemes involve the vehicle in mobility-related signaling hence effectively increasing the handover delay. Furthermore, these schemes do not properly address the advanced handover scenarios envisaged in future wireless networks. This paper, therefore, proposes a Logical Cross-Layer Optimization Block for VANET vertical mobility management framework utilizing cross-layer design, the IEEE 802.21 future
\end{abstract}


standard, and the recently emerged network-based localized mobility management protocol, Proxy Mobile IPv6, to further reduce handover delay.

Keywords: PMIPv6, MIH, MAG, LCO, VANET, Handover Latency

\section{Introduction}

Since wireless networks such as wireless LANs, IMT-2000, and Mobile WiMax introduced real-time multimedia services such as VoIP and interactive streaming, ubiquitous roaming support for real time multimedia traffic in an access independent manner becomes increasingly important. MIPv6 [1] specifies a host-based global mobility management scheme for an IP node to maintain network connectivity of a node as it roams around the Internet. MIPv6 performs three procedures sequentially to support handover: a movement detection, an address configuration, and a location update. These procedures have been shown to result in a long handover latency which strongly degrades the performance of packet transmission of mobile nodes (MNs). In order to reduce handover latency of MIPv6, IETF has standardized two schemes: Hierarchical MIPv6 (HMIPv6) [2], and fast handover for Mobile IPv6 (FMIPv6) [3]. Despite of lots of efforts, MIPv6 has not widely deployed, due to its heavy specification. That is, $\mathrm{MN}$ is required to implement complex specification which is difficult to deploy and maintain on small portable terminals with battery. Because of the deployment problem of MIPv6, network service providers and vendors have provided their own network-based mobility mechanisms within the localized area. However, these proprietary solutions are not interoperable with each other. Therefore, IETF recognized the need for standardization of network-based mobility services and IETF NetLMM (Network-based Localized Mobility Management) Working Group has discussed the problem statement [4] and goal [5] of network-based localized mobility management and has recently adopted Proxy Mobile IPv6 (PMIPv6) [6] as a single promising proposal for localized mobility standard.

Proxy Mobile IPv6 (PMIPv6) has been developed and applied successfully [7], [8], [9], [10], [11]. The PMIPv6 protocol does not require the MN to be participated in the IP mobility signalling required for handover procedure. The mobility access gateway (MAG) performs the IP mobility signalling required for handover procedure on behalf of the MN. However, since the MN moves between different two MAGs in PMIPv6, the handover latency cannot be avoided. Therefore, in order to reduce handover latency, various fast handover PMIPv6 mechanisms have been researched recently. Among them, to deal with heterogeneous wireless networks, several IEEE 802.21-assisted PMIPv6 mechanisms have been researched based on the exchange of IEEE 802.21 Media Independent Handover (MIH) related signalling messages as well as IP mobility related signalling messages [12], [13], [14], [15], [16], [17]. Although these approaches can reduce the handover latency through the interaction between cross layers, the MN has to implement MIH functionality. However, this can be inconsistent with the essential objective of the PMIPv6 that does not require the MN to be participated in the IP mobility required for handover procedure. In addition, the MIH functionality can be somewhat burdensome and power consumptive for MNs with limited 
resource and battery power. Moreover, there can be the number of MIH related signalling messages for handover procedure, which can cause considerable traffic overhead over wireless links as the number of MNs grows.

Therefore, to resolve the problem of existing mechanisms in [12], [13], [14], [15], [16], [17], an alternative IEEE 802.21-assisted PMIPv6 mechanism is proposed for fast vertical handover in heterogeneous wireless networks. The main objective of the proposed mechanism is to support fast vertical handover for the MN irrespective of the presence or absence of MIH functionality as well as IP mobility functionality, whereas the MN in existing mechanisms in [12], [13], [14], [15], [16], [17] has to implement MIH functionality. That is, the proposed mechanism does not require the MN to be participated in MIH related signalling required for handover procedure, which can be consistent with the essential objective of the PMIPv6. The base station (BS) with MIH functionality performs handover procedure on behalf of the MN. Therefore, the proposed mechanism can reduce burden and power consumption of MNs with limited resource and battery power. In addition, from analytical evaluation, experiment and simulation, the proposed mechanism can be shown to outperform the existing mechanism in terms of handover latency and total number of over-the-air signalling messages. Thus, the proposed mechanism can be expected to reduce the packet loss and the bandwidth consumption of wireless links during handover procedure comparing with the existing mechanism.

In this study we propose a unique logical assistive cross-layer design based PMIPv6 mobility protocol to support the vehicles continues connection while roaming between different network technologies (WiMax, LTE).

The proposed scheme introduces a novel solution for vertical handover support in PMIPv6 networks by which the MAG, on behalf of the vehicle, can maintain the vehicle's communication sessions during the handover process, irrespective of intra-domain or inter-domain handover. In this proposed scheme, the vehicle is still not aware of its movement when it moves in to another Local Mobility Anchor (LMA) domain (nLAM domain). Thus, the vehicle is not involved in any IP-mobility related signalling, regardless of its movement. When the vehicle moves into a new LMA domain, the new MAG will perform the correspondent registration on behalf of the vehicle. Accordingly, it can reduce latency and avoid host-based signalling. With this proposed scheme, the nature and advantageous characteristics of network-based mobility management of the PMIPv6 are retained, while still supporting inter-domain mobility management.

The rest of the paper is organized as follows; Section 2 provides the background and related work. In Section 3, we describe our proposed novel Inter-domain mobility support scheme. Section 4 estimates the performance of the proposed mechanism and the PMIPv6 mechanism through an evaluation model. Numerical results are given in Section 5. Finally, we conclude this paper in Section 6.

\section{Related works on Proxy Mobile IPv6}




\subsection{Proxy Mobile IPv6 (PMIPv6)}

Recently, Proxy Mobile IPv6 (PMIPv6) was standardized in Internet Engineer Task Force (IETF) [7] [8]. This protocol to supporting mobility does not require the mobile node $(\mathrm{MN})$ to be involved in the signalling required for mobility management. The mobility access gateway (MAG) in the network performs the signalling and does the mobility management on behalf of the MN. However, since the MN moves between different two MAGs in PMIPv6, the handover latency cannot be avoided. Therefore, the need to communicate efficiently on the move and to minimize the packet loss caused by a handover is becoming increasingly important because the handover latency is unacceptable for real-time IP services and throughput sensitive applications fig 1 presents PMIPv6 handover scheme.

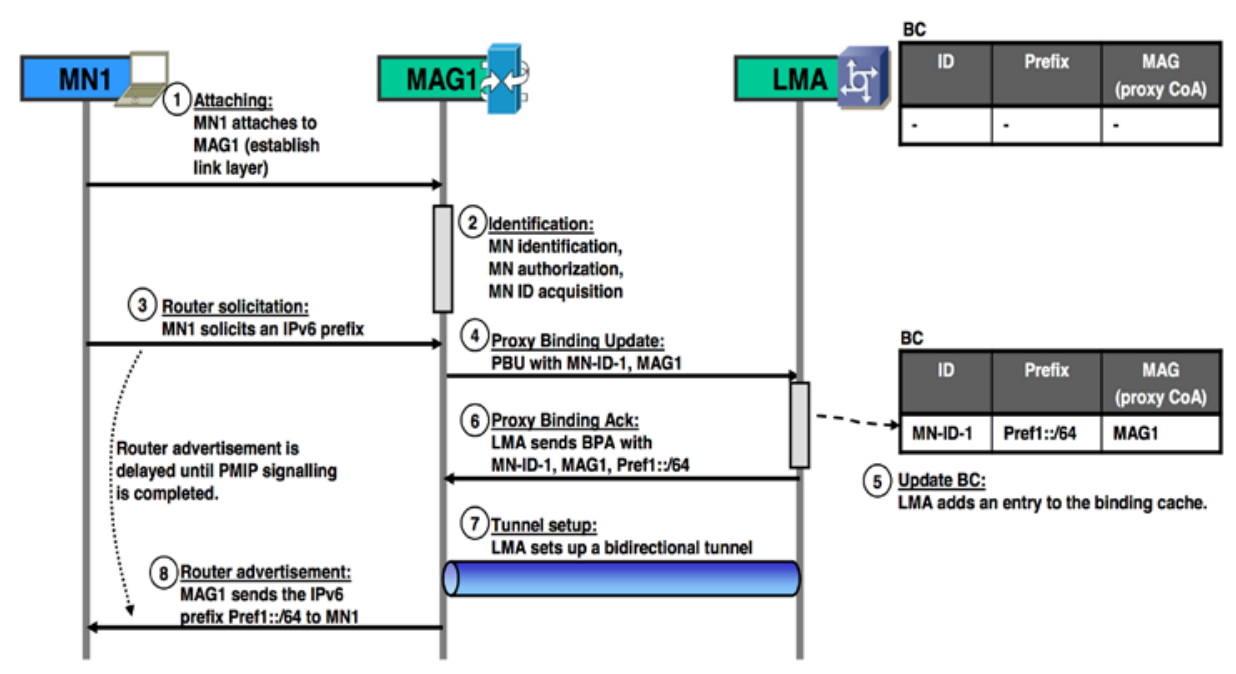

Figure 1 PMIPv6 Handover Scheme

To enhance the mobility support for the protocols many studies have been introduced, in [19], L. Banda, et al. Proposed a Vehicular Fast Mobile IPv6 (VFMIPv6) scheme in which each vehicle has a unique global IPv6 address assigned by the operator who manages the network. The vehicle keeps using this address while rooming from one RSU to another in the operator's domain. In this proposed method, the Current Access Router (CAR) responds immediately to the v-RtSolPr by sending a v-PrRtAdv without waiting for the Handover Acknowledgement (Hack) message from the TAR (Target Access Router). The reception of a HAck message triggered the forwarding of vehicles messages from the CAR to the TAR while the update of the binding in the vehicle's HA and the CNs is done by the CAR while sending a HI message to the TAR. In this scheme, the TAR transmits the vehicles cached packets upon the reception of the UNA (Unsolicited Neighbour Advertisement) message from the vehicle. This proposed method removes the delay imposed by the $\mathrm{DAD}$, by the construction of the global addresses for both the MNs and the ARs in the operator domain. Furthermore, this method doesn't use the FBU to initiate the Fast Handover nor the FBAck to put an end to it and allows to the PAR to send the HI message to the NAR just after sending the v-PrRtAdv to the MN. Also, unlike the Fast Handover for MIPv6 protocol, the Binding Update message is sent to HA/CNs soon in the handover process. In [30], Ahmed, et al. 
proposed a protocol which aims to reduce the scanning delay time during the handover process. The PRSU uses the velocity of the vehicle to estimate when this vehicle should start scanning and this minimizes the scanning delay. The PRSU participates also in the pre-selection and the preauthorization of the next RSU while the vehicle is still connected to the PRSU. In [20], A. Moravejosharieh et al. Early Binding Update Registration in PMIPv6 (EBRPMIPv6) In this protocol, the MAG has a pool of IP addresses assigned administratively and has also a table which contains information about its neighbouring MAGs.

Although these proposed solutions reduce the handover latency, the issue of vertical handover is still a challenging area that needs to be tackled.

\subsection{Media Independent Handover}

To provide a common solution for vertical handover in diverse access network environment, IEEE released 802.21 standard for handover function that does not depend on the media, which is known as Media Independent Handover (MIH) function [3], [4], [5]. The main objective of $\mathrm{MIH}$ function is to provide service abstraction to the upper layers using similar interface so it can improve the experience of the MN by supporting handovers in diverse network. This function helps the upper layer to keep the service running, service adjustments for different QoS, research on battery reliability, and network search and selection of links. In a network environment that is as diverse as the types 802 and cellular networks such as 3GPP, 3GPP2, MIH function can help upper layer to implement effective procedures for some of the services that can run on different networks. The point is, MIH function can be used as a facility for vertical handover in a varied wireless network environment [20].

\subsubsection{PMIPv6 Enabled MIH Protocol Integrated Schemes}

There are several schemes that benefits from the cross layer design of layer 2 (L2) MIH framework and layer 3 (L3) PMIPv6 protocol to optimize handover latency, packet loss or the overall handover performance. Hence, we classify the PMIPv6 and MIH integration as shown in Fig 2 and explain briefly the detailed handover operation using signalling flow diagram of PMIPv6 protocol assisted by MIH using the standard mechanism. The scenario proposed by [7] follows the handover procedure exactly as stated in the protocols drafts. When the serving wireless link signal becomes weak, the Media Independent Handover Function (MIHF) receives an event message about the signal strength of the serving link.

The MN attempts to search for other available neighbour networks by sending MIH_Get_Information request to MIIS server, which replies with MIH_Get_Information response. 


\section{Macrothink}

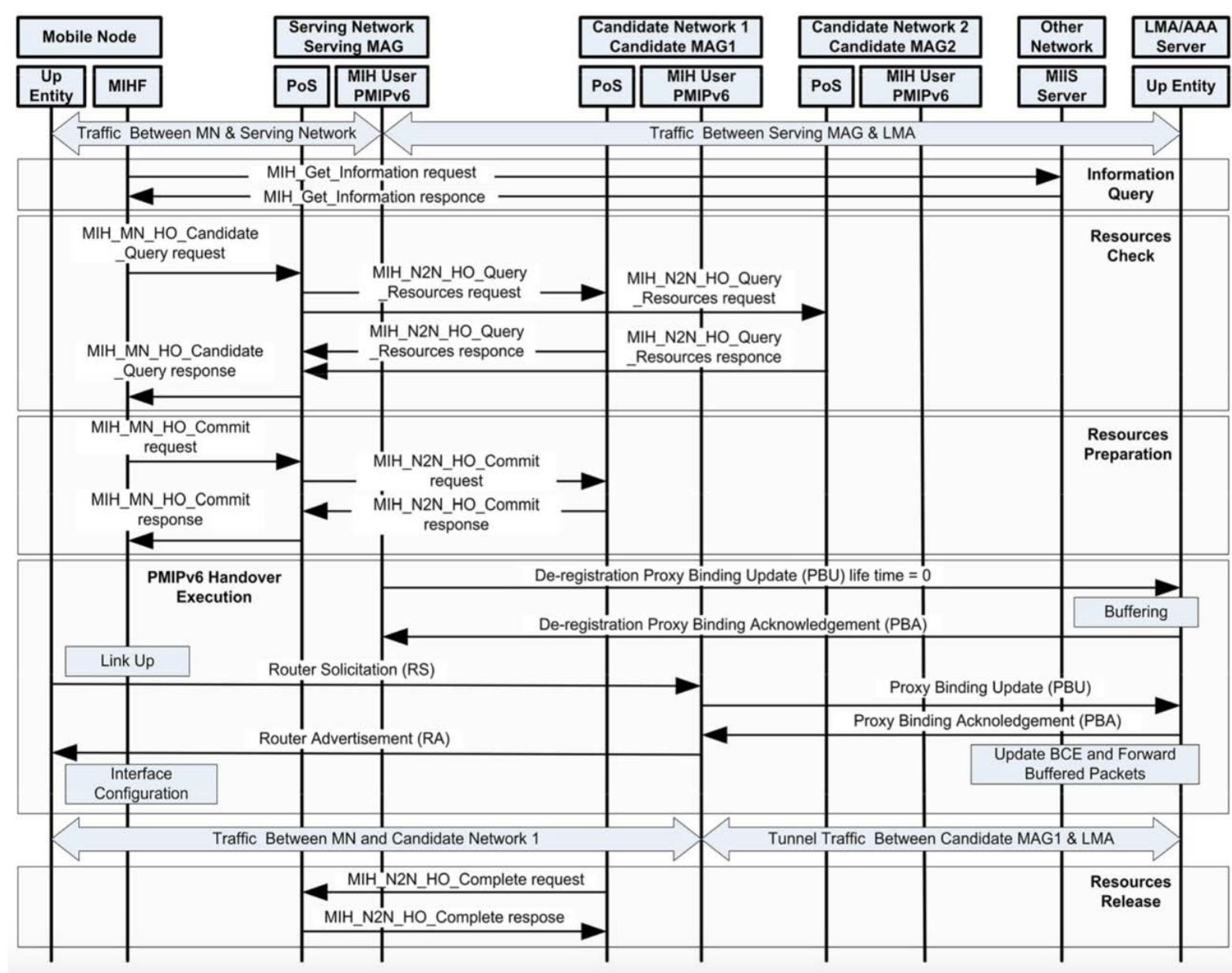

Figure 2 PMIPv6 Handover procedure assisted MIH

Based on the queried information, the MN determines the target candidate network and sends MIH_MN_HO_Commit request message to notify the serving network about the target network. The resources preparation occurs by sending MIH_N2N_HO_Commit request to the candidate target network, which then responds with MIH_N2N_HO_Commit to the serving network, which informs MN with MIH_MN_HO_Commit response.

During handover to the target network, the MN may detect the L2 detachment from the serving network. The serving network informs the LMA with deregistration PBU with lifetime set to zero and requests the LMA to buffer packets coming to the MN. The LMA then responds with PBA. Depending on the MN movements speed and the coverage area of the serving network, the detachment process with deregistration PBU/PBA with LMA may occur after $\mathrm{MN}$ attaches to the target network. The target network registers the $\mathrm{MN}$ location once it established L2 connection by sending PBA to the LMA which update their BCE and responds the PBA message and forward buffered packets to the MN. The target network can be solicited periodically or by router solicitation (RS) message from the MN and replies with RA message. After PMIPv6 handover is complete, the target network sends MIH_N2N_HO_Complete request message to the previous serving network which responds with MIH_N2N_HO_Complete response message. 


\section{Novel PMIPv6 Handover Method}

This section describes the novel vertical PMIPv6 handover scheme base vehicular environment, including the novel logical assistive cross-layer extension known as Logical Cross-Layer Optimization (LCO) Block. To support the vertical handover process, LCO Block is designed for PMIPv6 as shown in Fig 3, to support the handover process.

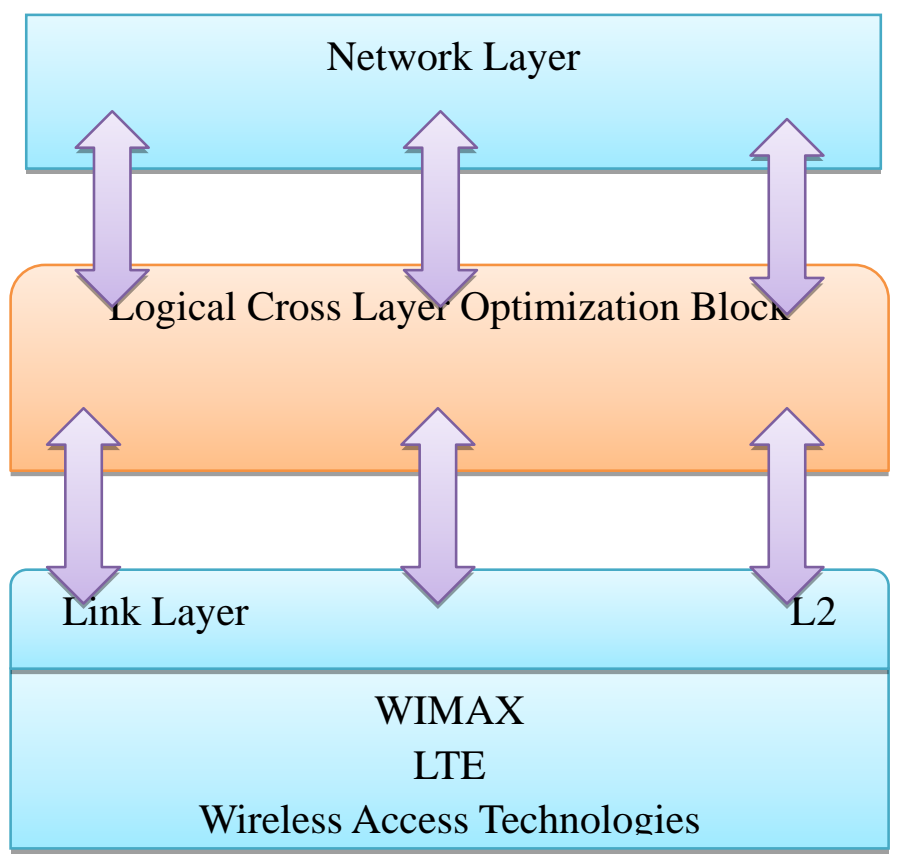

Figure 3. Logical Cross-Layer Block

The LCO block extension is introduced to solve the long handover latency issue for a vehicle roaming between two PMIPv6 domains (e.g. WiMax, LTE). For this extension we first define the Media Independent Information Service (MIIS) which is the core of LCO Block. Furthermore, this extension is assumed to be an intermediate layer between Layer 2 (L2) and Layer 3 (L3) that will exchange the information between L2, L3 stored within LCO Block. Fig 4 shows LCO Block.

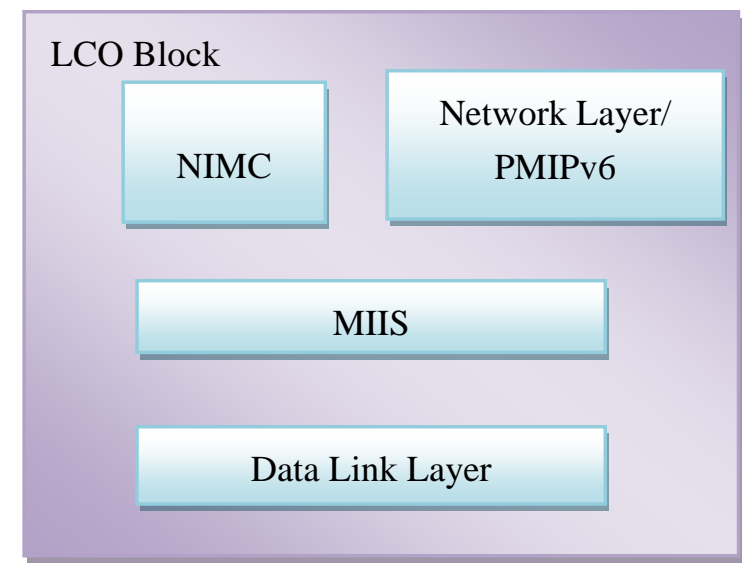

Figure 4 LCO Block 
The proposed LCO Block consists of:

1. Data Link Layer (L2) that provides information related to L2 signaling (signal strength).

2. MIIS, which continually scans the network for L2 and L3 information.

3. The information collected by MIIS will be encapsulated in the Network Information Management Container (NIMC), that will be used to evaluate the condition of the network and is it necessary to invoke the handover process or not.

The LCO Block will be used to extend the Mobile Access Gateway (MAG) since it is responsible of tracing the vehicles motion within the PMIPv6 domain. Fig 5 shows the proposed MAG extension.

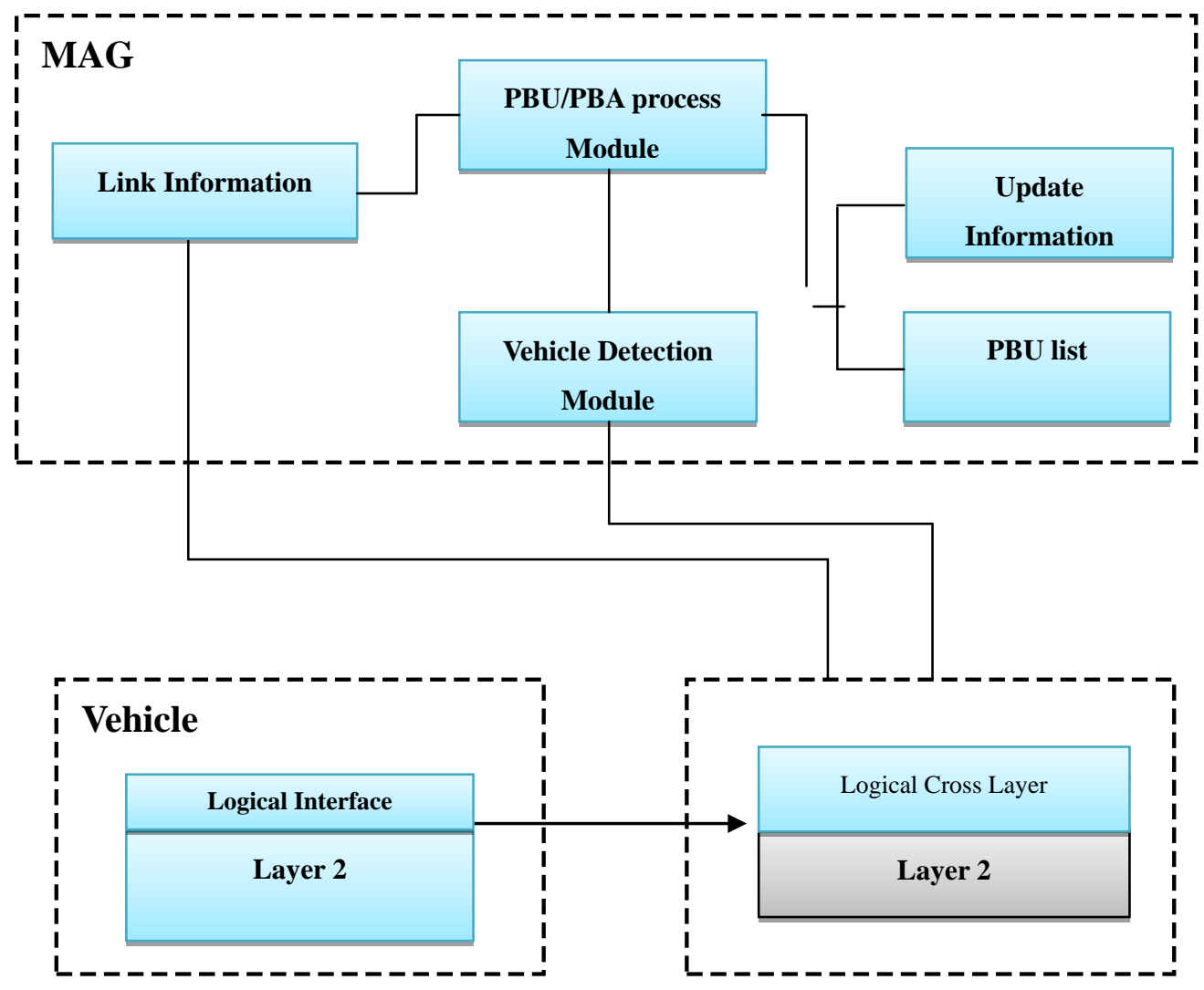

Figure 5 Proposed MAG Extension

As can be noticed from Fig 5 we extend the MAG operation by adding a new extension to the MAG design. Since the MAG knows the radio link information (i.e. MAC address and channel range of PoAs, etc.) of the PMIPv6 network domains from the pulled information stored in the LCO block. Hence, there will be no need to use the 'scanning' mechanism to find neighboring Base Station (BSs). In addition, L2 and L3 are assumed to start simultaneously this is because all the necessary information needed for the handover to start its process will be known as described. In other words, with the LCO block services, the vehicle and the PMIPv6 domain network entities, in particular the MAG in the access routers, 
are informed about the values of the relevant parameters (link going up, link going down, etc.) necessary in handover decision making prior to the actual handover process.

The process of the MAG extension can be summarized as:

1. When the MAG predicts the vehicle movement (before detachment/attachment) using the cross-layer information. If the received information contains new signalling information (L2 information), MAG will know that the vehicle is starting to move to a new domain. In such a case the MAG will send a fast BU message to its LMA in order to prevent any loss of packets during the handover process.

2. This early PBU message will trigger the LMA to estimate the vehicle detachment time earlier based on the information retrieved from the PBU message that provides the estimated based on the detection module block.

3. When receiving the extended PBU message LMA will use the analysed stored information to locate the address stored.

4. A pre-tunnel will be established between both LMAs to support the handover process.

5. After the pre-tunnel establishment, the vehicle will receive a PBA message form the new (nMAG1) to configure a new IPv6 address to its logical interface, the logical interface will enable the vehicle to configure two addresses.

The time needed for packet encapsulation will be discounted from the handover calculations because the process of encapsulation starts at the first stage of the handover execution. In other words, packet encapsulation process will start simultaneously with Data Link and Network Layer handover. Fig 6 shows the control flow for our proposed scheme. 


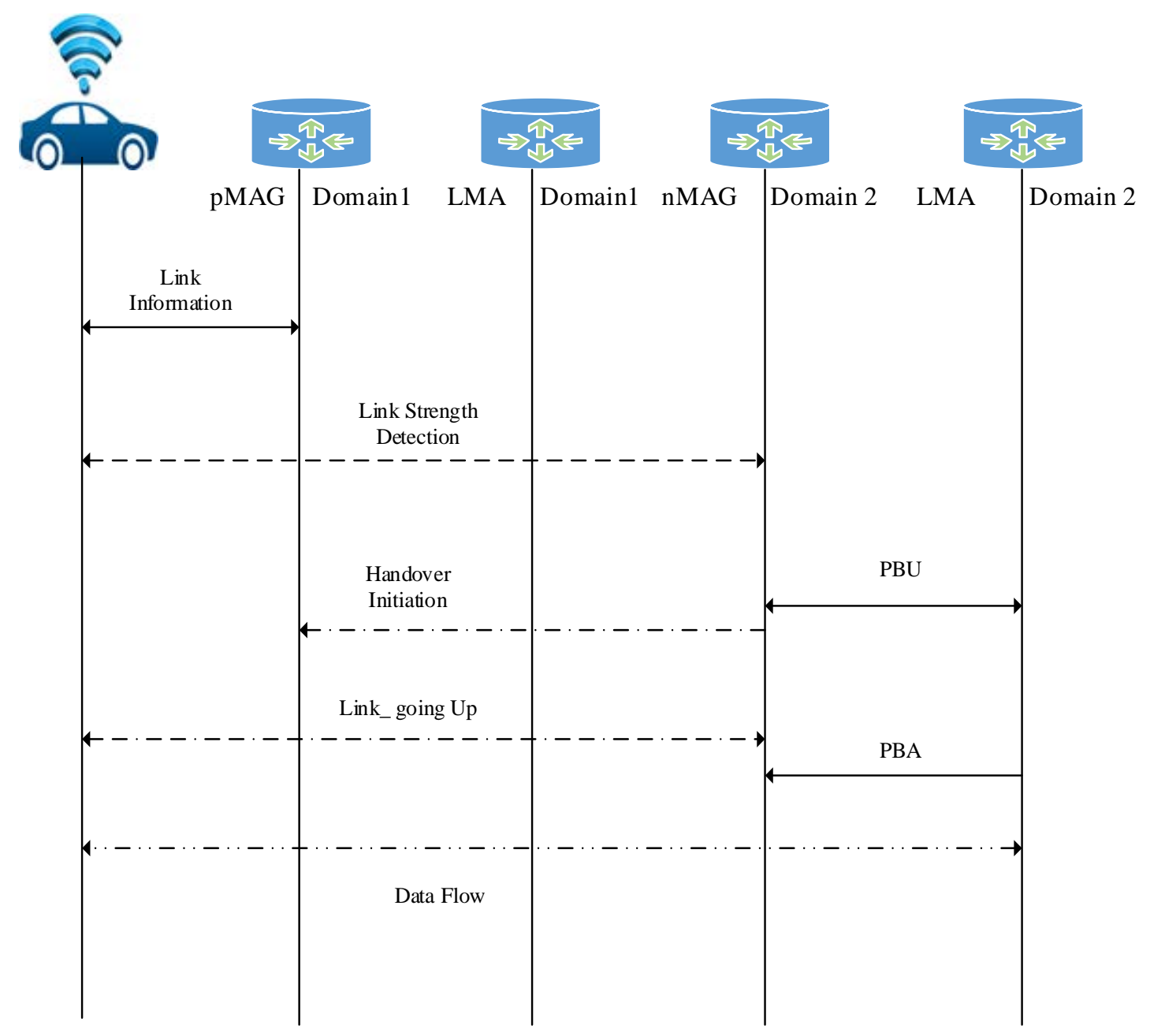

Figure 6 Novel PMIPv6 Handover Procedures

The novel PMIPv6 scheme exploits the services of the MIIS information contained in the NIMC to reduce handover delay, e.g., vertical handover delay which can cause significant delay.

The LCO Block enables some operations to be performed prior to the handover process while the vehicle is still connected to the old MAG's link in the First LMA domain. Thus, when the handover is eventually performed, there will be less delay causing procedures executed. For example, the vertical handover delay is dealt with by enabling the new next LMA to begin the handover procedure ahead of time by making use of the Cross Layer Optimization Block information and the. Utilizing the information, the vehicle and MAGs (pMAG and nMAG) get to know of their heterogeneous neighbouring networks' characteristics by requesting from information elements at a centralized information or MIIS server. The information server is assumed to be collocated within the LMA.

The Cross Layer Optimization Block information elements provides information that is essential for making handover estimation, such as, general information and accesses network specific information (e.g. network cost, service level agreements, QoS capabilities, etc.), 


\section{Macrothink}

point of attachment specific information (e.g. proxy care-of-address, data rates, MAC addresses, etc.), and other access network specific information.

\section{Performance Analysis}

In this study it is assumed that the vehicle maintains seamless connection while roaming between LMA domains.

\subsection{Network model}

Fig 7 shows the network model, which includes vehicle, WiMax and LTE Base Stations, WiMax MAG and LTE MAG, LMA. There are two LMA domains, WiMax LMA with pMAG1 and LTE LMA with nMAG1. The coverage of LMA is called domain, and the coverage of WiMax (BS) and LTE (BS) known as cells. In other words, each domain has $n$ cells. A (BS) connected to a MAG has a wireless interface for connecting vehicle (s). In this paper, we suppose that the vehicle is moving to a different LMA domain (WiMax LMA, LTE LMA).

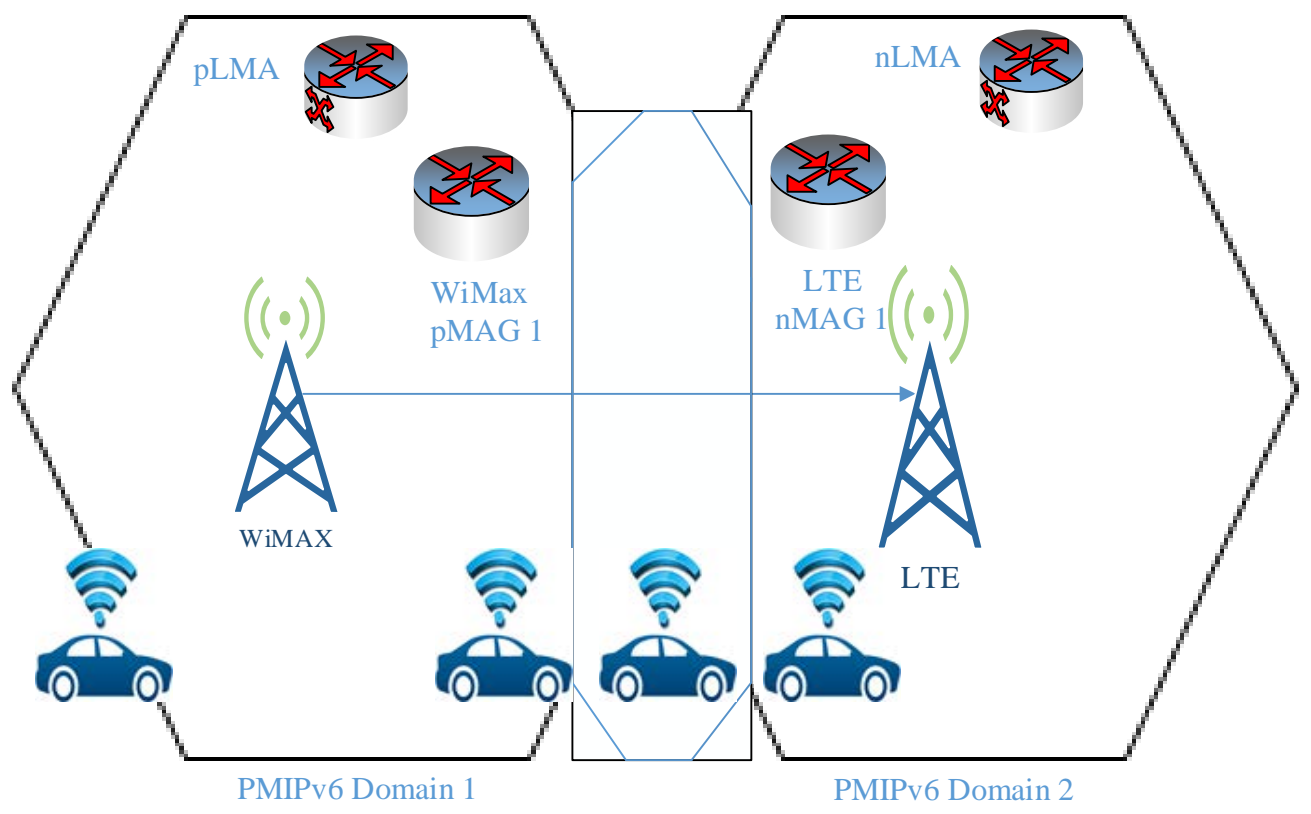

Figure 7 Proposed Network PMIPv6 Domain

Furthermore, we assume that once the vehicle passes the overlap area the LTE LMA will be able to intelligently calculate the stay time of the vehicle within the communication range of the first serving pMAG1 based on the LCO Block information.

We adopt the vehicle mobility model in where the direction of the vehicle motion in an LMA domain is uniformly distributed on $[0,2 \pi]$. 
For simplicity, we assume that the shape of the coverage area of a MAG is circular (non-circular areas, such as hexagonal shaped areas, can be reasonably approximated with the same size) and an inter-PMIPv6 consists of n MAGs with the same size of the coverage are of a $S_{A R}$ Vehicle (s) move at an average velocity of V.

Let $\mu_{c}, \mu_{d}$ be the cell crossing rate and domain crossing rate, respectively. Furthermore, let $\mu_{I}$ be the cell-crossing rate for that vehicle which is within the same PMIPv6 domain. Assuming that each AR has a coverage area of $S_{A R}$, the border crossing is given by [19].

$$
\begin{gathered}
\mu_{c}=\frac{2 v}{\sqrt{\pi S_{A R}}} \\
\mu_{d}=\frac{2 v}{\sqrt{\pi n S_{A R}}}=\frac{\mu_{c}}{\sqrt{n}} \\
\mu_{I}=\mu_{c}-\mu_{d}=\frac{\sqrt{n-1}}{\sqrt{n}} \mu_{c}
\end{gathered}
$$

The residence time in a cell and in a domain follows exponential distribution with parameters $\mu_{c}$ and $\mu_{d}$, while session arrival process follows a Poisson distribution with rate $\lambda_{s}$. Hence, the average number of cell crossing and domain crossing can be obtained as follows

$$
E\left(N_{d}\right)=\frac{\mu_{d}}{\lambda_{S}}
$$

In Equation (4) $\mathrm{E}\left(\mathrm{N}_{\mathrm{d}}\right)$ represents the vehicles crossing rate between the different LMAs. 


\subsection{Parameter Analysis}

We analyze the important performance metrics such as handover latency and binding update cost by using the network model and the mobility model. Handover latency has a significant impact on supporting real-time applications. In our novel PMIPv6 scheme, there is no need for movement detection and DAD. This is because the vehicle uses MN_pre address to uniquely configure its IP address. Furthermore, Layer 2 handover latency will be reduced because all the signaling information related with the connection going up and connection going down signals will be known in the early stages of the handover process. we assume that the binding update between the vehicles, HA and the CN does not occur within the inter-domain PMIPv6. Therefore, the handover latency for the novel scheme can be expressed in equation 5 :

$$
H_{\mathrm{L}}^{(\text {Proposed })}=\mathrm{T}_{\mathrm{L} 2}+\mathrm{T}_{\mathrm{L} 3}+\mathrm{T}_{\mathrm{RA}}
$$

where $H_{L}^{\text {(Proposed })}$ represents the handover latency for our scheme, $\mathrm{T}_{\mathrm{L} 2}, \mathrm{~T}_{\mathrm{L} 3}, \mathrm{~T}_{\mathrm{RA}}$ represents the time needed for layer 2, layer 3 and route acknowledgment time respectively. The binding update for the novel scheme can be expressed by equation 6 :

$$
P M I P v 6_{P B A}^{(P B U)}=T_{L 2}+2 * T_{\text {vehicle }, M A G}
$$

Where $T_{\text {vehiole, MAG }}$ represents the time needed for the vehicle to establish its connection.

\section{Numerical Results}

In this section, we use the parameters listed in Table 1 to calculate the handover latency for our novel solution. 
Table 1 Parameters used in the performance evaluation

\begin{tabular}{|l|l|l|}
\hline \multirow{5}{*}{ Delay } & Notation & Default Value \\
\cline { 2 - 3 } & PMIPv6 $_{\text {PBA }}^{(\text {PBU) }}$ & $30 \mathrm{~ms}$ \\
\cline { 2 - 3 } & $T_{L 2}$ & $200-400 \mathrm{~ms}$ \\
\cline { 2 - 3 } & $\mathrm{T}_{\mathrm{L} 3}$ & $50 \mathrm{~ms}$ \\
\cline { 2 - 3 } & $\mathrm{T}_{\mathrm{RA}}$ & $60 \mathrm{~ms}$ \\
\cline { 2 - 3 } & $2 T_{\text {vehiole, } M A G}$ & $10 \mathrm{~ms}$ \\
\cline { 2 - 3 } & Vehicle Speed & $50-150 \mathrm{~km}$ \\
\hline
\end{tabular}

We can notice that, the proposed PMIPv6 Scheme can reduce the handover latency time compared with other schemes introduced in [7] as shown in Fig 8 shows the handover latency for different speeds. Furthermore, Fig 9 shows the effect of the wireless link latency impact over the handover process. The results show that the proposed PMIPv6 reduces the overall handover latency compared with Modified-PMIPv6-MIH, PMIPv6-MIH and the Fast Approach schemes. Our proposed scheme showed improvements in different vehicle speeds and wireless link delay environments, this improvement will support the continues connection for the vehicle while roaming within a vertical domain (WiMax, LTE) LMA domains with the reduction of handover process time.

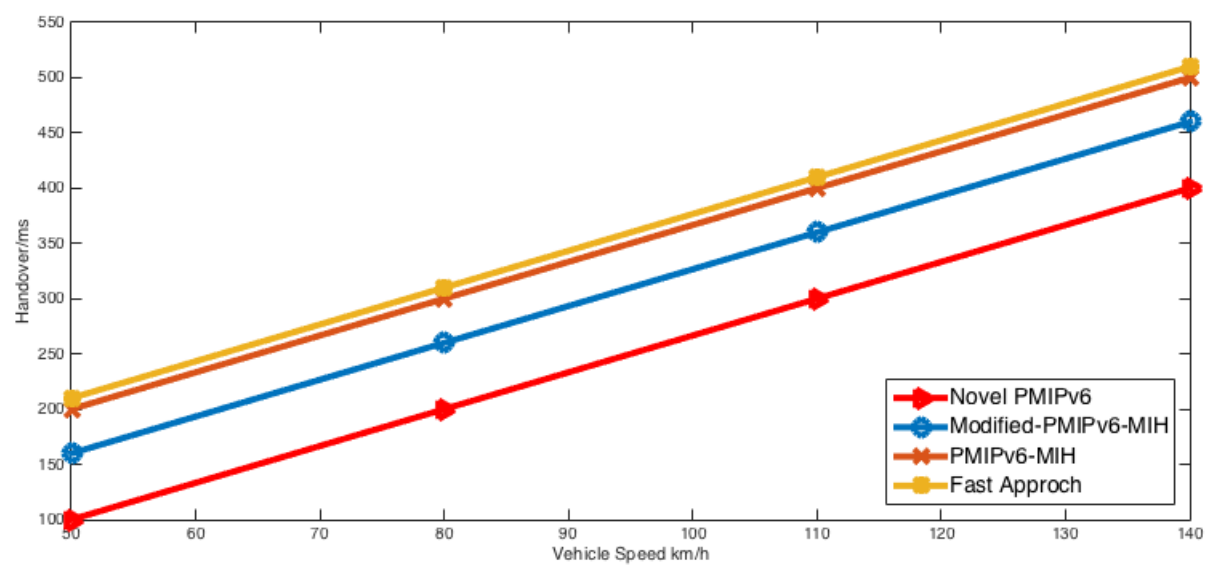

Figure 8 Proposed PMIPv6 Handover 


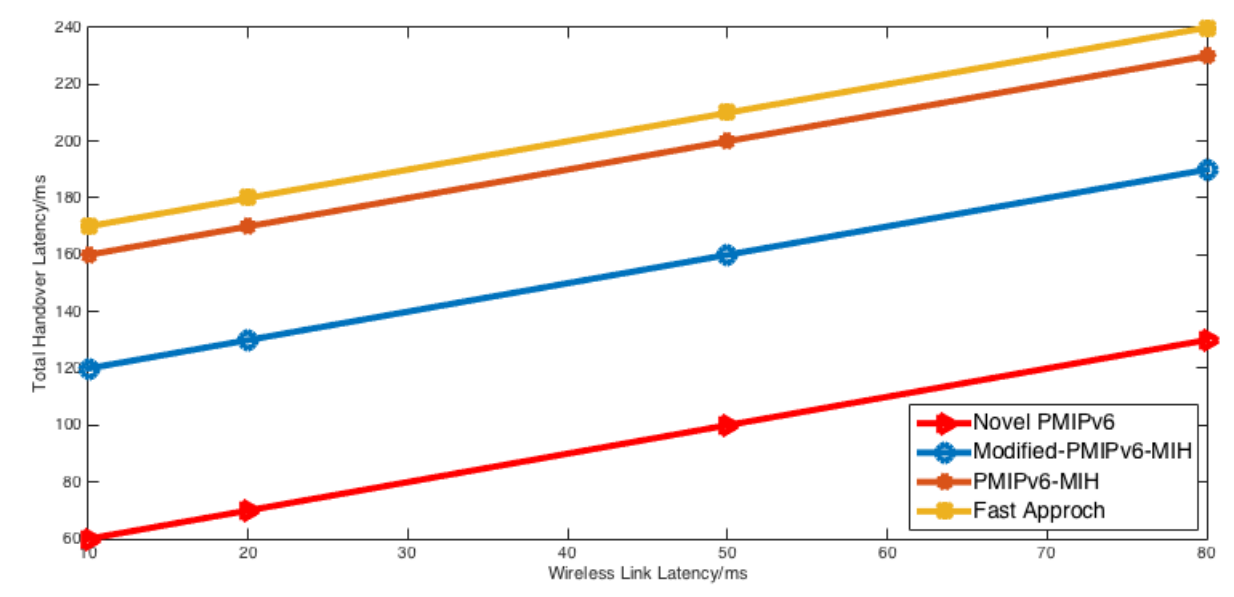

Figure 9 Wireless Link Delay

\section{Conclusion}

In this paper, we proposed a PMIPv6 Assistive Cross-Layer Design for vehicular environment to support continues roaming between (WiMax, LTE) LMA domains and compared our proposed technique with PMIPv6.

The proposed PMIPv6 scheme is based on LCO Block information function. Using the MIH services (MIIS), the MAG can obtain information without rout discovery or RtSolPr/PrRtAd messages. Thus, the handover latency time due to concurrent start of L2 and L3 handover process is reduced. In this way, our scheme is suitable for a cost-effective network compared with PMIPv6 scheme.

The novel PMIPv6 scheme is proposed to solve the high mobility of the vehicle while roaming between (WiMax, LTE) LMA domains by using a logical interface to support continuance communication within the new PMIPv6 LMA domain after completing the vertical PMIPv6 handover process.

Our future direction consists to developing both the simulation for the proposed scheme and the mathematical model to evaluate the novel PMIPv6 scheme in different environments for both inter-domain and intra-domain environments and compare the scheme to other related research studies. In addition, we will propose a new handover estimation technique. Further investigation of the novel intra-domain technique will be tested on a highway vehicular scenario. The impact of the intra-domain and inter-domain scheme on seamless connection support for vehicles roaming in PMIPv6 domains will be analytically analysed.

\section{References}

[1] Haider N. Hussain, Kamalrulnizam Abu Bakar and Shaharuddin Salleh "Using Media Independent Handover to Support PMIPv6 Inter-domain Mobility Based Vehicular Networks,” IJCNIS Vol. 4, No. 3, 2012. 
[2] Muhammad Zubair, Xiangwei Kong and Saeed Mahfooz, "Cross-layer Localized Mobility Management Based on SIP and HMIPv6 in Next Generation Networks,". Journal of Communications Vol. 9, No. 3, 2014. DOI: http://dx.doi.org/10.12720/jcm.9.3.217-225

[3] Seonggeun Ryu and Youngsong Mun, "Performance Analysis for FMIPv6 Considering Probability of Predictive Mode Failure," In Proc. of 2009 International Conference on Computational Science and Its Applications (ICCSA), 2009, pp. 34-38. DOI http://dx.doi.org/10.1109/ICCSA.2009.22

[4] J. Marquez-Barja, C. Calafate, J.-C. Cano, and P. Manzoni, "Evaluation of a technology-aware vertical handover algorithm based on the IEEE 802.21 standard," in Proc. of the 2011 IEEE Wireless Communications and Networking Conference, 2011, pp. 617-622. DOI http://dx.doi.org/10.1109/WCNC.2011.5779204

[5] F. Kronestedt, A. Furuskar, S. Landstrom, L. Falconetti, and K. Johansson, "Heterogeneous networks - Increasing cellular capacity,” Ericsson Reivew, vol. 88, no. 1, pp. 4-9, 2011.

[6] Gundavelli, S., Chowdhury, K., Devarapalli, V., Patil, B., Leung, K., et al.: Proxy mobile ipv6 (2008).

[7] Muayad Khalil Murtadha, Nor Kamariah Noordin, Borhanuddin Mohd Ali, "Survey and Analysis of Integrating PMIPv6 and MIH Mobility Management Approaches for Heterogeneous Wireless Networks,”. Springer Science, 2015, 1351-1376. DOI http://dx.doi.org/10.1007/s11277-015-2287-4

[8] K.-S. Kong, Y.-H. Han, M.-K. Shin, and H. You, "Mobility management for All-IP mobile networks: Mobile IPv6 vs. Proxy Mobile IPv6,” IEEE Wireless Communication, vol. 15, no. 2, pp. 36-45, 2008. DOI http://dx.doi.org/10.1109/MWC.2008.4492976

[9] H. Luo, H. Zhang, and Y. Q. V. Leung, “An approach for building scalable Proxy Mobile IPv6 domains," IEEE Trans. on Network and Service Management, vol. 8, no.3,pp.1932-4537, 2011.http://dx.doi.org/10.1109/TNSM.2011.071511.20100063

[10] S. Jeon and Y. Kim, "Cost-efficient network mobility scheme over Proxy Mobile IPv6 network,” IET Communications, vol. 5, no. 18, pp. 2656-2661, 2011. http://dx.doi.org/ $\underline{10.1049 / \text { iet-com.2011.0109 }}$

[11] J. Kim, R. Haw, E. Cho, C. Hong, and S. Lee, "A 6LoWPAN sensor node mobility scheme based on Proxy Mobile IPv6,” IEEE Trans. On Mobile Computing, vol. 11, no. 12, pp. 2060-2072, 2012. http://dx.doi.org/10.1109/TMC.2011.240

[12] L. A. Magagula and H. A. Chan, "IEEE 802.21-assisted cross layer design and PMIPv6 mobility management framework for next generation wireless networks,” in Proc. of the 2008 IEEE International Conference on Wireless \& Mobile Computing, Networking \& Communication, 2008, pp. 159-164. http://dx.doi.org/ 10.1109/WiMob.2008.46

[13] I. Kim, Y. C. Jung, and Y. Kim, "Low latency proactive handover scheme for Proxy MIPv6 with MIH,” Lecture Notes In Computer Science, vol. 5297, pp. 344 - 353, 2008. 
http://dx.doi.org/ 10.1007/978-3-540-88623-5_35

[14] S. Jeon, N. Kang, and Y. Kim, "Enhanced predictive handover for fast Proxy Mobile IPv6,” IEICE Trans. Commun., vol. E92-B, no. 11, pp. 3504-3507, 2009. http://dx.doi.org/ 10.1587/transcom.E92.B.3504

[15] H. Song, J. Kim, J. Lee, and H. S. Lee, “Analysis of vertical handover latency for IEEE 802.21-enabled Proxy Mobile IPv6,” in Proc. of the 13th International Conference on Advanced Communication Technology (ICACT), 2011, pp. 1059 - 1063.

[16] T. Melia, F. Giust, R. Manfrin, A. de la Oliva, C. J. Bernardos, and M. Wetterwald, "IEEE 802.21 and Proxy Mobile IPv6: A network controlled mobility solution,” in Proc. of the 2011 Future Network and MobileSummit Conference, 2011, pp. 1-8.

[17] J. Jose and A. Prithiviraj, "PMIPV6-HC-MIH: An approach for improving handover performance in NGWN," in Proc. of 2012 International Conference on Computing, Electronics and Electrical Technologies (ICCEET), 2012, pp. 910-914. http://dx.doi.org/10.1109/ICCEET.2012.6203737

[19] Xiao, L., \& Jiawei YANG, "Performance analysis of proxy mobile IPv6 based on IEEE802.16e,” Springer, 2010. http://dx.doi.org/10.1007/s11460-010-0112-0

[20] Gandeva B. Satrya, T. Brotoharsono and S. Wiranandi, "Performance Analysis of IEEE 802.21 MIH as a Function of Vertical Handover Using PMIPv6 and F-HMIPv6,” ICEC'15, 2015. DOI http://dx.doi.org/10.1145/2781562.2781565

\section{Copyright Disclaimer}

Copyright reserved by the author(s).

This article is an open-access article distributed under the terms and conditions of the Creative Commons Attribution license (http://creativecommons.org/licenses/by/3.0/). 